\title{
Impaired health-related quality of life in Graves' disease. A prospective study
}

\author{
T V Elberling ${ }^{1}$, A K Rasmussen ${ }^{2}$, U Feldt-Rasmussen ${ }^{2}$, M Hørding ${ }^{1}, \mathrm{H} \mathrm{Perrild}^{3}$ and G Waldemar ${ }^{1}$ \\ ${ }^{1}$ Memory Disorders Research Unit, Neuroscience Center, Copenhagen University Hospital, Rigshospitalet, Copenhagen, Denmark, ${ }^{2}$ Department of Medical \\ Endocrinology, Copenhagen University Hospital, Rigshospitalet, Copenhagen, Denmark and Department of Endocrinology, Bispebjerg Hospital, \\ Copenhagen, Denmark \\ (Correspondence should be addressed to G Waldemar; Email: gunhild.waldemar@rh.hosp.dk)
}

\begin{abstract}
Objective: In the acute, thyrotoxic phase, patients with Graves' disease often have both thyrotoxic and neuropsychiatric symptoms. The purpose of this prospective study was to examine healthrelated quality of life (HRQOL) in newly diagnosed and untreated Graves' patients and the effect of antithyroid medical treatment on HRQOL. In addition, we examined the potential influence of thyroid hormones and psychiatric symptoms on the impairment of HRQOL in the thyrotoxic phase.

Methods: A total of 30 consecutively referred patients with newly diagnosed and untreated Graves' disease and 34 age-, sex- and education-matched healthy volunteers were included in the study. HRQOL was assessed with the Medical Outcome Study 36-item Short-Form Health Status Survey (SF-36) before treatment, after reaching euthyroidism and 1 year after initiation of treatment. Results: In the thyrotoxic phase of Graves' disease, HRQOL was significantly impaired, in physical, mental and social dimensions. After reaching euthyroidism, the patients reported much fewer limitations on the subscales of SF-36. One year after initiation of treatment, all SF-36 scores had normalized. However, in some patients, HRQOL continues to be impaired even 1 year after initiation of treatment, as reviewed by the individual analysis. The reduced HRQOL in the acute phase of Graves' disease was correlated to depressive and anxiety symptoms, but thyroidassociated orbitopathy also influenced HLQOL.

Conclusions: Impaired HRQOL is common in the acute phase of Graves' disease. A significant proportion of the patients demonstrated persistent HRQOL impairment 1 year after initiation of treatment. Improvement of HRQOL in these patients remains a challenge for the clinician.
\end{abstract}

European Journal of Endocrinology 151 549-555

\section{Introduction}

Apart from the classical thyrotoxic symptoms (1), Graves' patients often have neuropsychiatric complaints in the acute phase of the disease, including memory problems, emotional lability, irritability and sometimes depressive, anxiety and hypomanic symptoms (2-4). Usually, the intensity of these complaints increases gradually for a long time before the patient is diagnosed with Graves' disease (5), and they may persist for an additional 3-6 months after treatment has been initiated. The complex of symptoms in the acute phase of Graves' disease and the duration of symptoms suggest that health-related quality of life (HRQOL) is affected in these patients. A few retrospective studies suggest that some patients have long-term neuropsychiatric symptoms despite successful antithyroid treatment $(6,7)$. Thus, the impact of Graves' disease on physical and social function, mental health and overall well-being of the patient might be an important outcome when treating patients with this disease. Several pervious studies, which focus on patients with thyroid-associated orbitopathy (TAO), have shown that TAO symptoms severely affect HRQOL in Graves' patients, even many years after treatment $(8-11)$. To our knowledge, HRQOL has never been examined in a prospective study including consecutively referred patients with untreated Graves' disease, without comorbidity, and without specific focus on Graves' patients with TAO. The purpose of this study was to examine HRQOL in newly diagnosed and untreated Graves' patients and the effect of antithyroid medical treatment on HRQOL. In addition, we examined the potential influence of thyroid hormones and psychiatric symptoms on the impairment of HRQOL in the thyrotoxic phase. 


\section{Subjects and methods}

This study included consecutively referred, newly diagnosed, and untreated patients with Graves' thyrotoxicosis, 18-60 years of age. The local ethics committee approved the study, and all participants gave their informed consent.

The diagnosis of Graves' disease was based on increased peripheral thyroid hormones, suppressed thyrotropin (thyroid-stimulating hormone (TSH)), positive TSH receptor antibodies (TRAb) and diffuse uptake on a Tc-99m pertechnetate scintigraphy of the thyroid gland. The laboratory variables (peripheral thyroid hormones, TSH and TRAb) were measured by standard methods. Patients with prior thyroid disease or psychiatric disorders were excluded from the study. Likewise, patients with neurologic disorders known to influence neuropsychiatric functions were excluded from the study. Patients unable to read Danish were also excluded. For comparison, 34 age-, sex- and education-matched healthy volunteers were recruited by advertising. Similar exclusion criteria were applied to the group of healthy volunteers, with the addition of exclusion upon family history of thyroid disease.

HRQOL was measured with the Danish version of the Medical Outcome Study 36-item Short-Form Health Status Survey (SF-36). The questionnaire includes 36 items that can be classified into the following eight health-status subscales: physical functioning, physical role limitations, bodily pain, general health perception, vitality, social functioning, emotional role limitations and mental health $(12,13)$. A standardized physical component score (PCS) and a standardized mental component score (MCS) were calculated (14). The PCS and MCS scores represent the deviation from the reference population (USA). The reference population has a mean PCS and MCS score of 50, with an S.D. of 10. The level of the Danish PCS and MCS is slightly higher than the US reference population level, but in order to improve comparability, the US reference population is used worldwide (13).

For examination of predictors for HRQOL in the acute thyrotoxic phase, the 17-item Hamilton Depression Rating Scale (HDRS) was used to quantify depressive symptoms (15), the 14-item Hamilton Anxiety Scale (HAS) was used to quantify anxiety symptoms (16, 17) and the 11-item Bech-Rafaelsen Mania Scale (BRMS) was used to quantify manic symptoms (18).

The degree of TAO was classified according to NOSPECS (19). (NOSPECS is a mnemonic composed of the first character describing classes of the ocular changes in Graves' disease, ie. No, Only, Soft, Proptosis, Exraocular, Corneal and Sight.) At each examination, all patients were classified into two groups according to NOSPECS: patients with no signs or symptoms of TAO (NOSPECS class zero) and patients with various degrees of TAO (NOSPECS classes 1-6).
The effect of treatment on HRQOL was examined in the Graves' patients after they reached euthyroidism and 1 year after initiation of treatment. The healthy volunteers were only examined once.

\section{Statistical analysis}

Statistical comparisons of thyroid hormones describing the effect of treatment in Graves' patients were performed with the paired-samples $t$-test. Statistical comparison of SF-36 measured in the toxic Graves' patients and healthy volunteers was performed with the Mann-Whitney U-test, and comparisons describing the effect of treatment in Graves' patients were performed with the Wilcoxon signed rank test. Statistical comparisons of the normally distributed PCS and MCS scores were performed with the unpaired two-tailed Student's t-test between Graves' patients and healthy volunteers and the paired-samples $t$-test between Graves' patients before and after treatment. In order to examine individual differences on the PCS and MCS, the percentage of patients who scored at least 2 S.D. below the control mean was calculated. To correlate SF-36 measures with psychiatric scales and thyroid hormone levels, Spearman's rank order correlation was used. All analyses were performed with the Statistical Products and Service Solutions, SPSS Version 11 (SPSS Inc., Chicago, IL, USA).

\section{Results}

Out of 53 consecutively referred patients with newly diagnosed and untreated Graves' disease, 30 were included in the study. The causes of exclusion were previous psychiatric disease $(n=5)$, ongoing drug abuse $(n=1)$, foreign primary language $(n=6)$, cerebral metastasis $(n=1)$, multiple sclerosis $(n=1)$, familial relation to the investigator $(n=1)$, vacation of the investigator $(n=1)$, no informed consent $(n=5)$, failure to return the questionnaire in more than one stage of the examination $(n=1)$ and lost to follow-up $(n=1)$. Basic characteristics are given in Table 1 .

In the acute thyrotoxic phase, patients reported more limitations on the subscales of SF-36 than the healthy volunteers, except for the subscale of bodily pain (Table 2). Additionally, the SF-36 component scores, PCS and MCS, were significantly lower in thyrotoxic patients than in the group of healthy volunteers.

Patients had significantly higher psychiatric scores as measured with HDRS and HAS. Most patients had mild to moderate depressive and anxiety symptoms. One patient was diagnosed with moderate/severe depression according to ICD-10, and antidepressive treatment was initiated. Psychiatric scales normalized after reaching euthyroidism and were also within the normal range at 1-year follow-up.

Higher score at the HDRS was significantly correlated to more limitations on the SF-36 subscales of 
Table 1 Basic characteristics of thyrotoxic Graves' patients and healthy volunteers.

\begin{tabular}{|c|c|c|c|c|}
\hline & \multirow[b]{2}{*}{ Healthy volunteers } & \multicolumn{3}{|c|}{ Graves' patients } \\
\hline & & Toxic & Euthyroid & 1-year \\
\hline $\operatorname{Sex}(F / M)$ & $30 / 4$ & $29 / 1$ & $26 / 1$ & $28 / 1$ \\
\hline Age (years) & $36.3 \pm 11.2$ & $35.8 \pm 10.0$ & & \\
\hline Education II & $15.2 \pm 1.8$ & $14.7 \pm 2.1$ & & \\
\hline Psychiatric disposition (yes/no) & $4 / 30$ & $4 / 26$ & & \\
\hline Hamilton Depression Rating Scale & $0(0-4)$ & $8(2-14)$ & & \\
\hline Hamilton Anxiety Scale & $0(0-5)$ & $11(2-19)$ & & \\
\hline Bech-Rafaelsen Mania Scale & $0(0-0)$ & $2(0-8)$ & & \\
\hline Duration from initial investigation (months) & & & $3.9 \pm 1.5$ & $12.7 \pm 0.7$ \\
\hline Monitoring of thyroid function (times per year) & & & & $10(7-17)$ \\
\hline TAO ('NOSPECS' class 0/classes 1-6) & $34 / 0$ & $21 / 9$ & $21 / 6$ & $20 / 9$ \\
\hline Thyrotropin (TSH) $(0.4-4.0 \mathrm{mIU} / \mathrm{l})$ & $1.5(0.7-2.5)$ & $0.01(<0.01-0.07)$ & $0.01(<0.01-3.38)^{\star *}$ & $0.01(<0.01-7.22)^{\# \# \#}$ \\
\hline Total thyroxine $(60-160 \mathrm{nmol} / \mathrm{l})$ & $95 \pm 16$ & $255 \pm 63$ & $107 \pm 27^{* * *}$ & $111 \pm 24^{\# \# \#}$ \\
\hline Free thyroxine $(9.1-23.8 \mathrm{pmol} / \mathrm{l}$ & $12.5 \pm 1.3$ & $45.6 \pm 11.9$ & $13.7 \pm 4.2^{\star * *}$ & $13.7 \pm 2.5^{\# \# \# / \S}$ \\
\hline Total triiodothyronine $(0.9-2.7 \mathrm{nmol} / \mathrm{l})$ & $1.5 \pm 0.3$ & $5.4 \pm 1.5$ & $1.8 \pm 0.5^{\star \star \star}$ & $1.5 \pm 0.4^{\# \# \#}$ \\
\hline
\end{tabular}

Education II = primary education (max. 12 years) plus vocational training (max. 5 points). Psychiatric scores are given as median (range). Number of thyroid function monitoring visits within the 1 -year follow-up period; values are given as median (range). TAO = thyroid-associated orbitopathy. TAO was classified according to 'NOSPECS'. Thyrotropin (TSH): in brackets $=95 \%$ reference limits. Values are given as median (range). Peripheral thyroid hormones: in brackets $=95 \%$ reference limits. Values are given as mean \pm S.D. ${ }^{* \star *} P<0.001$ Graves' patients in the toxic phase versus euthyroid phase (paired-samples $t$-test). ${ }^{* *} P<0.01$ Graves' patients in the toxic phase versus euthyroid phase (Wilcoxon signed rank test). ${ }^{\# \# \#} P<0.001$ Graves' patients in the toxic phase versus 1-year after initiation of treatment (paired-samples $t$-test). §28 patients in statistical analysis.

physical functioning, physical role limitations, general health, social functioning and emotional role limitations, as well as lower scores on the PCS and MCS. A higher score on the HAS was significantly correlated to patients reporting more limitations on the SF-36 subscale of mental health, as well as lower scores on the MCS. No correlations were found between SF-36 measures and the level of thyroid hormones.

After the initial investigation had been completed, all patients received methimazole treatment, but due to side effects (itching), three patients were changed to propylthiouracil.

At euthyroid follow-up, a 59-year-old woman dropped out of the study because she felt overburdened, one patient failed to return the questionnaire, and one patient was subclinically hypothyroid upon examination. After reaching euthyroidism, the remaining 27 patients reported much fewer limitations on the subscales of SF-36, although the general health perception subscale was similar to that of the toxic level, and the social functioning subscale was improved but not significantly different from the toxic level (Table 2; Fig. 1). Although the eight subscales of SF-36 returned to normal after euthyroidism was reached, a greater variation was identified within the group of euthyroid patients. Regarding the standardized PCS and MCS, at euthyroid follow-up, PCS was still impaired, when comparing euthyroid patients with healthy volunteers, whereas MCS had normalized. By 1 year after initiation of treatment, all SF-36 scores had normalized.

In the individual analysis of the PCS, 63\% of the patients scored at least 2 S.D. below the control mean in the toxic phase, $33 \%$ in the euthyroid phase, and $14 \%$ at 1-year follow-up. Concerning the individual analysis of the MCS, $43 \%$ of the patients scored at least 2 S.D. below the control mean in the toxic phase, $15 \%$ in the euthyroid phase, and $11 \%$ at 1 -year follow-up. In seven patients, the PCS or MCS score was at least 2 s.D. below the control mean in the toxic phase as well as at 1-year follow-up.

At each examination, Graves' patients were classified into two groups according to NOSPECS: one group with no signs or symptoms of TAO and one group with various degrees of TAO. Graphical illustration reveals a general trend toward TAO's influencing SF-36 measures (Fig. 2). Statistical analysis of the SF-36 measures yielded no significant differences between the two groups, except that the subscale of general health perception in the toxic phase was significantly more impaired in patients with TAO. Almost all of the patients with TAO had mild symptoms and were treated with local measures (such as lubricating eye-drops). In only one patient did the severity of TAO result in treatment with anti-inflammatory and immunomodulatory treatment. None had orbital radiation or surgical therapy.

\section{Discussion}

To our knowledge, this is the first prospective study including consecutively referred patients with untreated Graves' disease, without comorbidity, and without specific focus on Graves' patients with TAO. In the thyrotoxic phase of Graves' disease, patients had impaired quality of life, in physical, mental and social dimensions. Following diagnosis and treatment, the SF-36 subscores generally improved, but in some patients HLQOL remained impaired, as reviewed by the standardized component scores, PCS and MCS. Lower SF-36 scores 


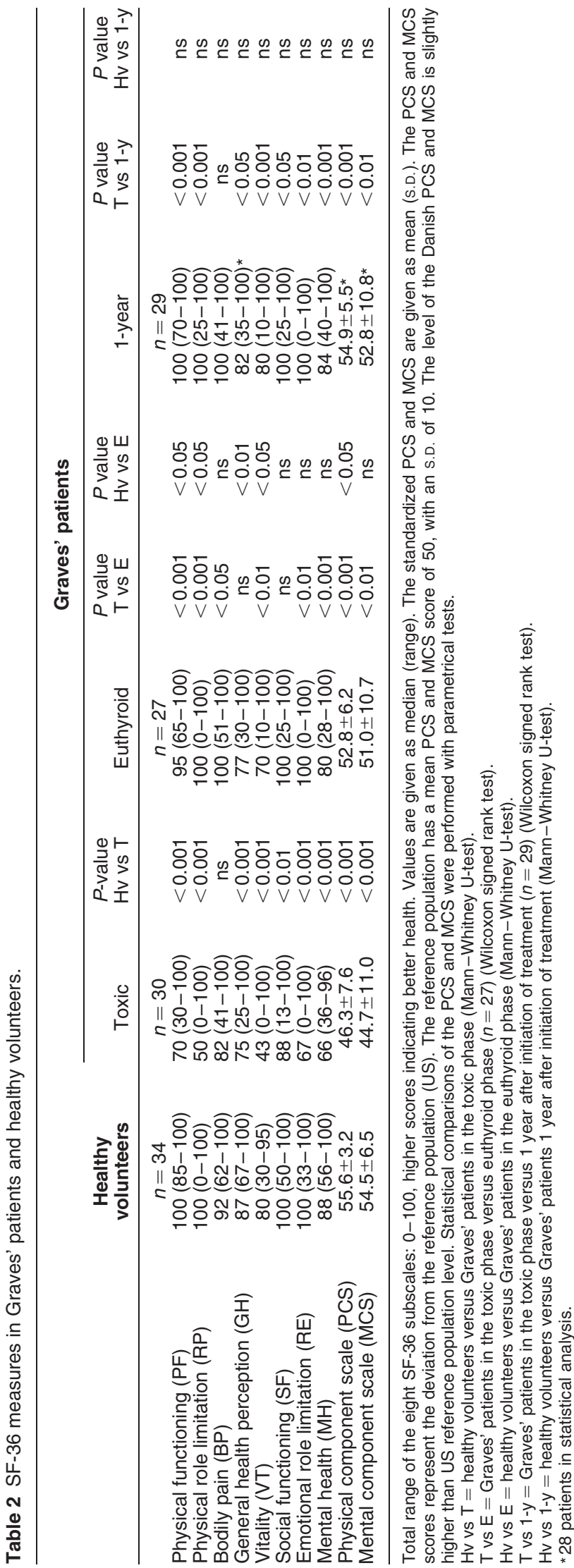

in the toxic phase correlated significantly with the level of depressive and anxiety symptoms.

The SF-36 is a multipurpose survey of general health status examining physical and mental wellbeing as well as the ability to function in everyday life. SF-36 enables group comparisons of generic health concepts (20). SF-36 was chosen in the present study because it is a short, multi-item scale with acceptable validity and low respondent burden as compared to the more comprehensive surveys. Shortform scales are likely to have problems of ceiling and floor effects, but the summary measures of SF-36 (PCS and MCS) are not associated with these problems. Furthermore, through the International Quality of Life Assessment Project, the SF-36 has been adapted for international conditions, making comparisons between various nationalities and language versions possible $(21-23)$.

One prospective study, examining sickness-related behavior with the Sickness Impact Profile (SIP) in 14 hyperthyroid Graves' patients, found dysfunction in especially three categories of the SIP (sleep and rest, home management, and leisure, pastimes and recreation). Treatment resulted in a 'relatively prompt resolution of the patients' dysfunctional behaviour' (24). The SIP is a well-known measure of HRQOL, but this study of 14 hyperthyroid patients included some with other chronic disorders (such as depression, obesity and congestive heart failure) known to affect HRQOL and therefore compromising the interpretation of the results. However, the study supports the main finding of the present study that HRQOL is reduced in the acute phase of Graves' thyrotoxicosis.

We have shown that although the SF-36 subscales normalized after 1 year of antithyroid medical treatment, the standardized component scores remained impaired in some patients. Thus, in 14\% of the patients, PCS was at least 2 S.D. below the control mean at 1 year after initiation of treatment; in $11 \%$ of the patients, MCS was at least 2 S.D. below the control mean at 1 year after initiation of treatment. The fact that residual HRQOL complaints might persist after normalization of the thyroid dysfunction supports the findings of a few retrospective questionnaire studies that thyroid disease patients have lower ratings of HRQOL even years after diagnosis (25-27).

Several previous reports dealing specifically with Graves' TAO patients have shown that TAO symptoms severely affect HRQOL of Graves' patients, even many years after treatment $(8-11)$. Our study found no statistically significant differences in SF-36 measures between the two subgroups of patients with and without TAO, except that the subscale of general health perception in the toxic phase was significantly more impaired in patients with TAO. However, the study revealed a general trend toward TAO influencing SF-36 measures. At 1 year after initiation of treatment, seven patients had PCS and MCS scores at 


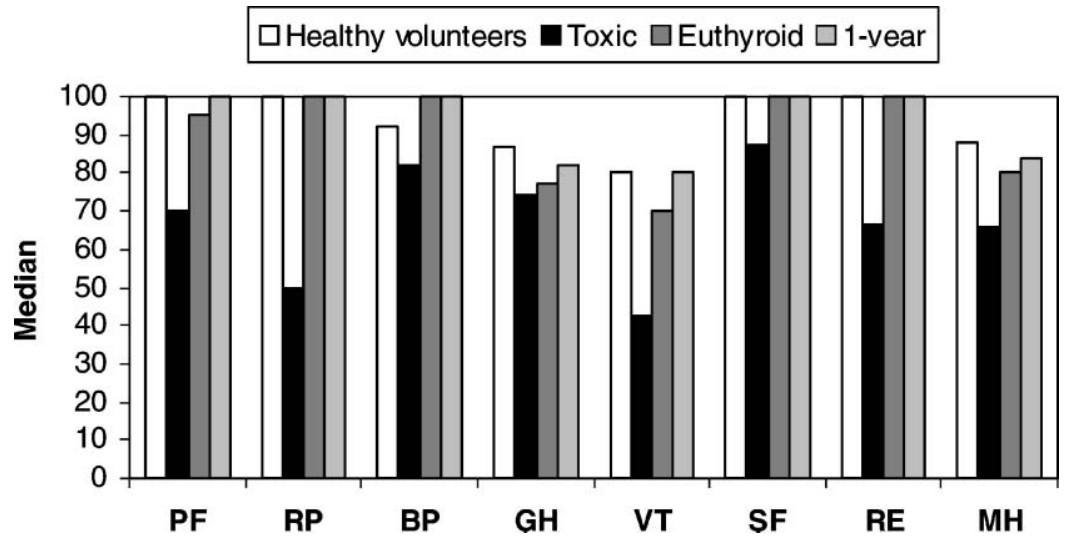

a

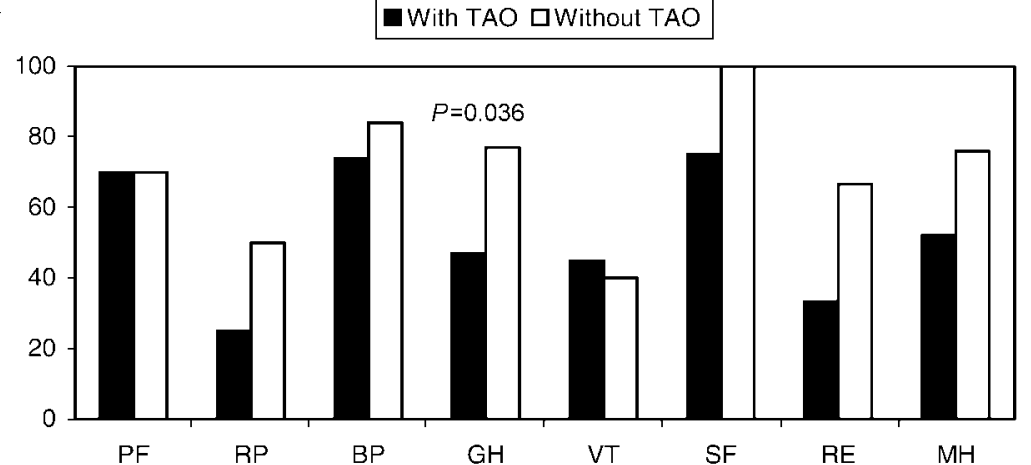

b

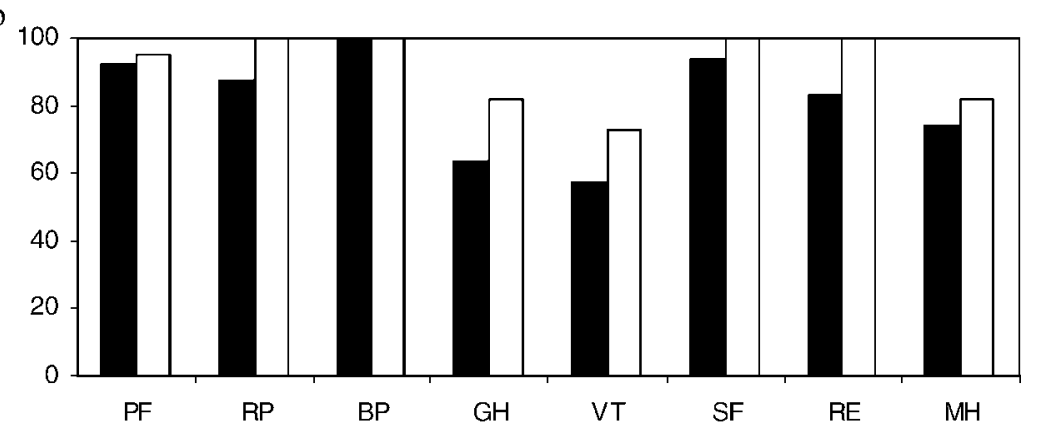

c

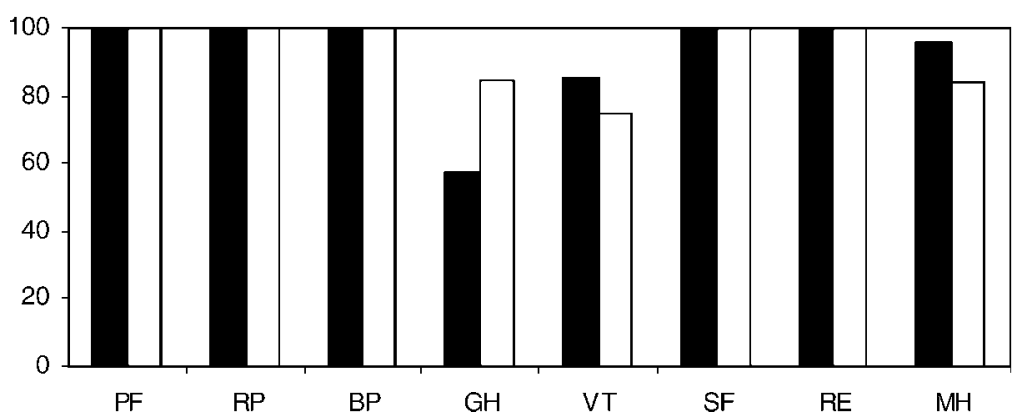

Figure 1 SF-36 measures in Graves' patients as compared to healthy volunteers. The eight SF-36 measures are presented as median scale scores in healthy volunteers and Graves' patients in toxic phase, after reaching euthyroidism, and 1 year after initial investigation. Higher scores indicate better health. PF = physical functioning, $\mathrm{RP}=$ physical role limitations, $\mathrm{BP}=$ bodily pain, $\mathrm{GH}=$ general health perception, VT = vitality, SF = social functioning, $\mathrm{RE}=$ emotional role limitations, $\mathrm{MH}=$ mental health.

Figure 2 SF-36 measures in Graves' patients with and without TAO in (a) toxic phase,

(b) euthyroid phase, and (c) 1 year after initial investigation. $\mathrm{PF}=$ physical functioning, $\mathrm{RP}=$ physical role limitations, $\mathrm{BP}=$ bodily pain, $\mathrm{GH}=$ general health perception, $\mathrm{VT}=$ vitality, $\mathrm{SF}=$ social functioning, $\mathrm{RE}=$ emotional role limitations, $\mathrm{MH}=$ mental health, $\mathrm{PCS}=$ physical component scale, MCS = mental component scale. The SF-36 measures are presented as median scale scores in Graves' patients. Higher scores indicate better health.

least 2 S.D. below the control mean, and only one of these patients had severe treatment requiring TAO. The fact that almost all patients with TAO had only mild symptoms might explain the relatively moderate effect of TAO on HRQOL. This study revealed no other clear explanation of why HRQOL remained impaired in some patients.

Graves' thyrotoxicosis is a disease which has gradually affected the life of the patients, usually for many months, but sometimes years, prior to the diagnosis. 
Even subclinical hyperthyroid patients (suppressed TSH with normal peripheral thyroid hormones) have been shown to have significantly lower PCS and MCS than matched controls (28). Since the decreased HRQOL in the acute phase of Graves' thyrotoxicosis most likely reflects the level of thyrotoxic and neuropsychiatric manifestations at this point, improvement of these symptoms should result in normalization of HRQOL. After normalization of the thyroid function, most of the previous studies have shown that the thyrotoxic and neuropsychiatric manifestations of Graves' disease have almost disappeared $(2-4)$, as was also confirmed by the present study. Thus, the impaired HRQOL in some patients after successful antithyroid treatment, as seen in the present study, is not exclusively related to the thyroid disease. In addition to TAO, the HRQOL impairment might also be influenced by still unknown factors or may be related to individual differences in perception of disease. Thus, for example, psychologically vulnerable individuals might have prolonged HRQOL impairment despite successful antithyroid treatment. This has to be examined in large prospective studies.

The symptoms related to Graves' disease affected patients to such an extent that the HRQOL of these patients was impaired. In some patients, HRQOL remained reduced 1 year after initiation of treatment, suggesting that the personal and social consequences may be greater than previously assumed. Thus, not only thyroid function but also HRQOL needs to be addressed as clinical outcomes when treating patients with Graves' disease.

\section{Acknowledgements}

We thank the Danish Medical Research Council, the 1991 Pharmacy Foundation, and the Health Insurance Fund for financial support to the Memory Disorders Research Unit. We also acknowledge an additional grant from the Danish Hospital Foundation for Medical Research, Region of Copenhagen, the Faroe Islands and Greenland. We are grateful for the work of laboratory technician Betty Fischer.

\section{References}

1 Trzepacz PT, Klein I, Roberts M, Greenhouse J \& Levey GS. Graves' disease: an analysis of thyroid hormone levels and hyperthyroid signs and symptoms. American Journal of Medicine $1989 \mathbf{8 7}$ 558-561.

2 Kathol RG, Turner R \& Delahunt J. Depression and anxiety associated with hyperthyroidism: response to antithyroid therapy. Psychosomatics 198627 501-505.

3 Trzepacz PT, McCue M, Klein I, Greenhouse J \& Levey GS. Psychiatric and neuropsychological response to propranolol in Graves' disease. Biological Psychiatry 198823 678-688.

4 Paschke R, Harsch I, Schlote B, Vardarli I, Schaaf L, Kaumeier S, Teuber J \& Usadel KH. Sequential psychological testing during the course of autoimmune hyperthyroidism. Klinische Wochenschrift $199068942-950$.
5 Sonino N, Girelli ME, Boscaro M, Fallo F, Busnardo B \& Fava GA. Life events in the pathogenesis of Graves' disease. A controlled study. Acta Endocrinologica 1993128 293-296.

6 Perrild H, Hansen JM, Arnung K, Olsen PZ \& Danielsen U. Intellectual impairment after hyperthyroidism. Acta Endocrinologica (Copenhagen) $1986112185-191$.

7 Bommer M, Eversmann T, Pickardt R, Leonhardt A \& Naber D. Psychopathological and neuropsychological symptoms in patients with subclinical and remitted hyperthyroidism. Klinische Wochenschrift $1990 \mathbf{6 8} 552-558$.

8 Gerding MN, Terwee CB, Dekker FW, Koornneef L, Prummel MF \& Wiersinga WM. Quality of life in patients with Graves' ophthalmopathy is markedly decreased: measurement by the medical outcomes study instrument. Thyroid 1997 7 885-889.

9 Egle UT, Kahaly GJ, Petrak F, Hardt J, Batke J, Best J \& Rothenbacher M. The relevance of physical and psychosocial factors for the quality of life in patients with thyroid-associated orbitopathy (TAO). Experimental and Clinical Endocrinology and Diabetes 1999107 S168-S171.

10 Kahaly GJ, Hardt J, Petrak F \& Egle UT. Psychosocial factors in subjects with thyroid-associated ophthalmopathy. Thyroid 2002 12 237-239.

11 Terwee C, Wakelkamp I, Tan S, Dekker F, Prummel MF \& Wiersinga W. Long-term effects of Graves' ophthalmopathy on health-related quality of life. European Journal of Endocrinology $2002146751-757$.

12 Ware JE Jr \& Sherbourne CD. The MOS 36-item short-form health survey (SF-36). I Conceptual framework and item selection. Medical Care 199230 473-483.

13 Bjorner JB, Damsgaard MT, Watt T, Bech P, Rasmussen NK, Kristensen TS, Modvig J, Thundeborg K. Danish SF-36 Manual. Copenhagen: Lif, 1997.

14 Ware JE, Koskinski M, Keller SD. SF-36 Physical and Mental Health Summary Scales: A User's Manual. Boston, MA: 1994.

15 Hamilton M. Development of a rating scale for primary depressive illness. British Journal of Social and Clinical Psychology 19676 278-296.

16 Hamilton M. The assessment of anxiety states by rating. British Journal of Psychiatry 195932 50-55.

17 Hamilton M. Diagnosis and rating of anxiety. British Journal of Psychiatry 19693 76-79.

18 Bech P, Bolwig TG, Kramp P \& Rafaelsen OJ. The Bech-Rafaelsen Mania Scale and the Hamilton Depression Scale. Acta Psychiatrica Scandinavica $1979 \mathbf{5 9} 420-430$.

19 Werner SC. Modification of the classification of the eye changes of Graves' disease: recommendations of the Ad Hoc Committee of the American Thyroid Association. Journal of Clinical Endocrinology and Metabolism $1977 \mathbf{4 4} 203-204$.

20 Ware JE Jr. The SF-36 Health Survey. In Quality of Life and Pharmacoeconomics in Clinical Trials, 2nd edn, ch 33, pp 337-345. Ed. B Spilker. Philadelphia: Lippincott-Raven, 1996.

21 Keller SD, Ware JE Jr, Bentler PM, Aaronson NK, Alonso J, Apolone G, Bjorner JB, Brazier J, Bullinger M, Kaasa S, Leplége A, Sullivan M \& Gandek B. Use of structural equation modeling to test the construct validity of the SF-36 Health Survey in ten countries: results from the IQOLA Project. International Quality of Life Assessment. Journal of Clinical Epidemiology $1998 \mathbf{5 1}$ 1179-1188.

22 Keller SD, Ware JE Jr, Gandek B, Aaronson NK, Alonso J, Apolone G, Bjorner JB, Brazier J, Bullinger M, Fukuhara S, Kaasa S, Leplége A, Sanson-Fisher RW, Sullivan M \& WoodDauphinee S. Testing the equivalence of translations of widely used response choice labels: results from the IQOLA Project. International Quality of Life Assessment. Journal of Clinical Epidemiology 199851 933-944.

23 Raczek AE, Ware JE, Bjorner JB, Gandek B, Haley SM, Aaronson NK, Aaronson NK, Apolone G, Bech P, Brazier JE, Bullinger M \& Sullivan M. Comparison of Rasch and summated rating scales constructed from SF-36 physical functioning items in seven countries: results from the IQOLA Project. International Quality 
of Life Assessment. Journal of Clinical Epidemiology $1998 \mathbf{5 1}$ $1203-1214$

24 Rockey PH \& Griep RJ. Behavioral dysfunction in hyperthyroidism. Improvement with treatment. Archives of Internal Medicine $19801401194-1197$.

25 Berg G, Michanek A, Holmberg E \& Nystrom E. Clinical outcome of radioiodine treatment of hyperthyroidism: a follow-up study. Journal of Internal Medicine 1996239 165-171.

26 Torring O, Tallstedt L, Wallin G, Lundell G, Ljunggren JG, Taube A, Sääf M \& Hamberger B. Thyroid Study Group. Graves' hyperthyroidism: treatment with antithyroid drugs, surgery, or radioiodine - a prospective, randomized study. Thyroid Study Group. Journal of Clinical Endocrinology and Metabolism $1996 \mathbf{8 1} 2986-2993$.
27 Fahrenfort JJ, Wilterdink AM \& van der Veen EA. Long-term residual complaints and psychosocial sequelae after remission of hyperthyroidism. Psychoneuroendocrinology 200025 201-211.

28 Biondi B, Palmieri EA, Fazio S, Cosco C, Nocera M, Sacca L, Filetti S, Lombardi G \& Perticone F. Endogenous subclinical hyperthyroidism affects quality of life and cardiac morphology and function in young and middle-aged patients. Journal of Clinical Endocrinology and Metabolism $2000 \mathbf{8 5} 4701-4705$.

Received 12 April 2004

Accepted 18 August 2004 